\title{
Potential use of lenvatinib for patients with unresectable hepatocellular carcinoma including after treatment with sorafenib: Real-world evidence and in vitro assessment via protein phosphorylation array
}

\author{
Tetsu Tomonari, ${ }^{1,}$, Yasushi Sato ${ }^{2, *}$, Hironori Tanaka ${ }^{1}$, Takahiro Tanaka ${ }^{1}$, Yasuteru \\ Fujino ${ }^{1}$, Yasuhiro Mitsui ${ }^{1}$, Akihiro Hirao ${ }^{1}$, Tatsuya Taniguchi ${ }^{1}$, Koichi Okamoto ${ }^{1}$, \\ Masahiro Sogabe ${ }^{1}$, Hiroshi Miyamoto ${ }^{1}$, Naoki Muguruma ${ }^{1}$, Harumi Kagiwada ${ }^{3}$, \\ Masashi Kitazawa ${ }^{4}$, Kazuhiko Fukui ${ }^{3,4}$, Katsuhisa Horimoto ${ }^{3,4}$ and Tetsuji Takayama ${ }^{1}$ \\ ${ }^{1}$ Department of Gastroenterology and Oncology, Institute of Biomedical Sciences, Tokushima University Graduate School, \\ Tokushima, Japan \\ ${ }^{2}$ Department of Community Medicine for Gastroenterology and Oncology, Tokushima University Graduate School of Biomedical \\ Sciences, Tokushima, Japan \\ ${ }^{3}$ Molecular Profiling Research Center for Drug Discovery, National Institute of Advanced Industrial Science and Technology, \\ Tokyo, Japan \\ ${ }^{4}$ SocIUM, Inc., Tokyo, Japan \\ *These authors contributed equally to this work \\ Correspondence to: Tetsuji Takayama, email: takayama@tokushima-u.ac.jp \\ Keywords: hepatocellular carcinoma; lenvatinib; sorafenib; hepatic reserve function; FGFR \\ Received: April 02, 2020 \\ Accepted: June 01, 2020 \\ Published: June 30, 2020 \\ Copyright: Tomonari et al. This is an open-access article distributed under the terms of the Creative Commons Attribution License 3.0 (CC \\ BY 3.0), which permits unrestricted use, distribution, and reproduction in any medium, provided the original author and source are credited.
}

\section{ABSTRACT}

The efficacy and safety of lenvatinib (LEN) as a second/third-line treatment for unresectable hepatocellular carcinoma (HCC) after sorafenib (SOR) therapy remains unknown. We evaluated the outcomes of second/third-line LEN treatment, investigated the sensitivity of a SOR-resistant HCC cell line (PLC/PRF5-R2) to LEN, and assessed their signal transduction pathways by protein array analysis. We retrospectively enrolled 57 patients with unresectable HCC. Fifty-three radiologically evaluated patients comprised 34 molecular-targeted agent (MTA)-naive (first-line), nine intolerant to SOR (second-line), and 10 resistant to regorafenib (third-line). The objective response rates (ORRs) were $61.8 \%$ in first-line, $33.3 \%$ in second-line, and $\mathbf{2 0 . 0 \%}$ in third-line groups. The overall survival (OS) in the first-line was significantly longer than that in the third-line group $(p<0.05)$. Patients with better liver functional reserves (child score, ALBI grade) exhibited higher ORR and longer OS. The IC ${ }_{50}$ of LEN against PLC/PRF5-R2 was significantly higher than that against PLC/PRF5. LEN significantly inhibited more LEN-related signal transduction pathways in PLC/PRF5 than in PLC/PRF5-R2 cells. This suggests that LEN is active and safe as a second/ third-line treatment for unresectable HCC. LEN seems more effective for patients with HCC with better hepatic reserve functions or before MTA-resistance is acquired because of the partial cross-resistance to SOR.

\section{INTRODUCTION}

Hepatocellular carcinoma (HCC) is reportedly the fifth most commonly-diagnosed malignancy and the second leading cause of cancer-related death worldwide [1]. For patients with unresectable advanced HCC, sorafenib (SOR) was the first recommended systemic therapy to demonstrate a survival benefit with an adequate safety profile $[2,3]$. SOR is an oral molecular-targeted agent (MTA) that blocks RAF kinase, vascular endothelial growth factor (VEGF) receptors, and the platelet-derived growth factor (PDGF) receptors KIT and fms-related 
tyrosine kinase 3 (FLT3). A phase III SHARP trial showed a median overall survival (mOS) of 10.7 months and a disease control rate (DCR) of $43 \%$ in the SOR treatment group of unresectable HCC patients with well-preserved liver function. However, the benefits of SOR were not sustained as the median time-to-progression (mTTP) was only 5.5 months. Subsequently, a randomized, placebocontrolled, phase III RESORCE trial reported that regorafenib (REG), an oral MTA, resulted in survival benefits for patients with advanced HCC who were progressing while on SOR. In this trial, REG showed a 2.8-month improvement in mOS, with a $38 \%$ reduction in the risk of death [4]. Thus, REG has been the standard second-line chemotherapy for patients refractory to SOR. However, the frequency of advanced HCC patients for whom REG is indicated is reportedly only $30.6-37 \%$ [5-8], and more than half of these patients are not able to receive second-line treatment. Recently, a phase III REACH-2 trial demonstrated that ramucirumab (RAM) in advanced HCC patients with baseline $\alpha$-fetoprotein levels $\geq 400 \mathrm{ng} / \mathrm{ml}$ after SOR failure showed significant improvements in mOS [8.5 vs. 7.3 months; hazard ratio: $0.710 ; 95 \%$ confidence interval (CI): 0.531-0.949]. However, the patients for whom RAM is indicated are limited to a proportion of only $23.3 \%$ [9].

Furthermore, cabozantinib (CAB), an oral MTA for MET, VEGFR2, and RET, was shown to be superior than the placebo in a phase III CELESTIAL trial (mOS: 10.2 vs. 8.0 months; hazard ratio: 0.76 ; 95\% CI: $0.63-0.92$ ) of advanced HCC patients with SOR resistance. In this study, a relatively high frequency of Grade 3 or 4 adverse events $(68 \%)$ was observed after CAB treatment, such as palmar-plantar erythrodysesthesia (17\%), hypertension $(16 \%)$, and increased aspartate aminotransferase levels $(12 \%)$ [10].

A recent phase III REFLECT trial indicated that lenvatinib (LEN) was not inferior to SOR as a first-line treatment for unresectable HCC [11]. LEN is an oral MTA that targets VEGF receptors 1-3, FGF receptors 1-4, PDGF receptor $\alpha$, RET, and KIT [12-16]. The REFLECT trial showed a mOS of 13.6 months and an mTTP of 8.9 months, where the objective response rate (ORR) was $40.6 \%$ for patients of the LEN group. Thus, LEN has been approved in Japan and other countries as a first-line systemic treatment for patients with unresectable advanced HCC [11]. Due to the promising efficacy, tolerability, and cost-effectiveness of LEN [17], it has been used not only as a first-line treatment but also as a second-line treatment for patients intolerant to SOR and as a third-line treatment following SOR and REG failure in clinical practice. However, there have only been a few reports regarding the efficacy and adverse effects of LEN when used as a second- or third-line treatment for advanced HCC $[18,19]$. Especially, little is known about the clinical characteristics of HCC patients that receive potential therapeutic benefits from second- or third-line LEN treatment.
Currently it is not evident whether LEN or SOR should be used as the first-line therapy for advanced HCC. However, both drugs are similar MTAs, and some components of the target molecules (VEGFR, PDGFR, KIT) are common to both agents. Therefore, it is highly plausible that they might generate cross resistance to each other. In this context, it is expected that the efficacy of LEN as a second- or third-line treatment for HCCs, beyond SOR, could be less than that of LEN as a firstline treatment. Moreover, it is unclear which signal transduction pathways are associated with the efficacy of LEN against HCC cells that acquire SOR resistance.

Accordingly, we evaluated the characteristics, therapeutic efficacy, and safety of LEN as a second- and third-line treatment and also as a first-line treatment for unresectable HCC patients in clinical practice. Moreover, to expand upon these clinical findings in vitro, we assessed the anti-tumor activity of LEN using a SOR-resistant cell line and performed a comprehensive phosphorylated protein array analysis associated with 377 signal transduction pathways using SOR-resistant and parental HCC cells.

\section{RESULTS}

\section{Patient characteristics}

A total of 57 patients with unresectable HCC who had received LEN were enrolled in this study. However, of these, four patients were excluded from the analysis because they could not be evaluated using modified Response Evaluation Criteria in Solid Tumors (mRECIST) measurements due to renal failure. Thus, 53 patients were retrospectively analyzed. Baseline characteristics of these patients are listed in Table 1 . The median observation period following the initiation of treatment with LEN was 266 (111-603) days. The median age of the patients was 71 years (range, 47-85 years). Of all patients, 14 (26.4\%) were HBV antigen-positive and 22 (41.5\%) were HCV antibody-positive and the male population was apparently larger than the female cohort.

The Eastern Cooperative Oncology Group performance status (ECOG PS) was 0 in 48 patients (90.6\%). The median alpha-fetoprotein (AFP) value was $37 \mathrm{ng} / \mathrm{ml}$ (range 2-568100) and Child-Pugh scores before treatment were 5 points in 30 patients and 6 points in 23 patients. ALBI grades before treatment were 1 point in 22 patients and 2 points in 31 patients. LEN therapy was initiated at Barcelona Clinic Liver Cancer (BCLC) stage $\mathrm{B}$ in 37 patients and at stage $\mathrm{C}$ in 16 patients. The median number of cases of transarterial chemoembolization (TACE) before treatment with LEN was 1 (0-9). Among the 53 patients, 34 were MTA-naive (first-line), nine were intolerant to SOR (second-line), and 10 were resistant to SOR and REG (third-line). The median duration of followup in each group was as follows: 255 (118-603) days for first-line, 391 (111-603) days for second-line, and 265 
Table 1: Characteristics of patients with unresectable hepatocellular carcinoma treated with lenvatinib

\begin{tabular}{|c|c|c|c|c|c|}
\hline Characteristics & $\begin{array}{l}\text { All } \\
(n=53)\end{array}$ & $\begin{array}{l}\text { First-line } \\
(n=34)\end{array}$ & $\begin{array}{l}\text { Second-line } \\
(n=9)\end{array}$ & $\begin{array}{l}\text { Third-line } \\
(n=10)\end{array}$ & $p$-value \\
\hline Age, median [range], (years) & $71[47-85]$ & $72[53-85]$ & $70[61-80]$ & $66[47-83]$ & 0.45 \\
\hline Sex (male/female), $n$ & $44 / 9$ & $30 / 4$ & $7 / 2$ & $7 / 3$ & 0.38 \\
\hline Etiology (HBV/HCV/NBNC), $n$ & $14 / 22 / 17$ & $10 / 12 / 12$ & $2 / 4 / 3$ & $2 / 6 / 2$ & 0.87 \\
\hline ECOG PS $(0 / 1), n$ & $48 / 5$ & $32 / 2$ & $7 / 2$ & $10 / 0$ & 0.24 \\
\hline Platelets, median [range], $\left(10^{4} / \mu \mathrm{l}\right)$ & $14.4[6.2-31.8]$ & $14.4[6.2-31.8]$ & $16.3[9.0-25.7]$ & $11.4[6.2-23.7]$ & 0.45 \\
\hline M2BpGi [range] (C. O. I) & $1.54[0.44-13.1]$ & $1.25[0.48-5.76]$ & $1.59[0.44-13.1]$ & $2.23[0.58-5.4]$ & 0.17 \\
\hline Child-Pugh score $(5 / 6 / 7 / 8), n$ & $30 / 23 / 0 / 0$ & $22 / 12 / 0 / 0$ & $5 / 4 / 0 / 0$ & $3 / 7 / 0 / 0$ & 0.15 \\
\hline ALBI Grade $(1 / 2 / 3), n$ & $22 / 31 / 0$ & $13 / 21 / 0$ & $4 / 5 / 0$ & $1 / 9 / 0$ & 0.08 \\
\hline Number of intrahepatic lesions (None/1/2-7/> 7) & $0 / 6 / 23 / 24$ & $0 / 4 / 17 / 13$ & $0 / 1 / 4 / 4$ & $0 / 1 / 2 / 7$ & 0.70 \\
\hline $\begin{array}{l}\text { Maximum size of intrahepatic lesion (None/ } \leq \\
50 />50)(\mathrm{mm})\end{array}$ & $0 / 42 / 11$ & $0 / 26 / 8$ & $0 / 7 / 2$ & $0 / 9 / 1$ & 0.79 \\
\hline Portal vein invasion (absent/present), $n$ & $47 / 6$ & $30 / 4$ & $7 / 2$ & $9 / 1$ & 0.72 \\
\hline Extrahepatic spread (absent/present), $n$ & $42 / 11$ & $29 / 5$ & $5 / 4$ & $8 / 2$ & 0.16 \\
\hline AFP, median [range] $(\mathrm{ng} / \mathrm{ml})$ & $37[2-568100]$ & $12[2-568100]$ & $414[4-2262]$ & $37[4-5050]$ & 0.73 \\
\hline BCLC stage $(\mathrm{B} / \mathrm{C}), n$ & $37 / 16$ & $24 / 10$ & $5 / 4$ & $8 / 2$ & 0.51 \\
\hline Previous treatment times of TAE/TACE [range] & $1[0-9]$ & $1[1-6]$ & $2[0-9]$ & $2[1-6]$ & 0.10 \\
\hline Initial dose of Lenvatinib (12/8/4), (mg), $n$ & $30 / 22 / 1$ & $23 / 10 / 1$ & $3 / 6 / 0$ & $4 / 6 / 0$ & 0.13 \\
\hline
\end{tabular}

Abbreviations: HBV, hepatitis B virus; HCV, hepatitis C virus; NBNC, non B non C; ECOG PS, Eastern Cooperative Oncology Group performance status; M2BPGi, mac-2 binding protein glycosylation isomer; ALBI, albumin-bilirubin; AFP, alpha-fetoprotein; BCLC, Barcelona Clinic Liver Cancer; TAE/TACE, transcatheter embolization/chemoembolization.

(132-507) days for third-line. There were no significant differences in patient characteristics between those with and without a previous history of MTA treatment, including hepatic reserve function and tumor burden.

\section{Response to LEN}

Fifty-three patients had measurable lesions that could be evaluated by enhanced computed tomography/ magnetic resonance imaging (CT/MRI) at 8 weeks after the initiation of LEN treatment. Of these 53 patients, two exhibited a complete response (CR) (3.8\%), 24 had a partial response (PR) (45.3\%), 25 had stable disease (SD) $(47.2 \%)$, and two presented with progressive disease (PD) (3.8\%). The ORR and DCR were $49.1 \%$ $(26 / 53)$ and $96.2 \%(51 / 53)$, respectively (Supplementary Table 1). Regarding the response in each treatment-line group, ORRs in the first-line group $(61.8 \% ; 21 / 34)$ were higher than those in the second-line group $(33.3 \%, 3 / 9 ; p$ $=0.28)$ and those in the third-line group $(20.0 \%, 2 / 10 ; p$ $=0.27$; Table 2). Moreover, the ORR with BCLC stage B $(20 / 37,54.1 \%)$ was higher than that with BCLC stage C $(6 / 16,37.5 \%)$. In terms of hepatic reserve functions, the ORR in the Child-Pugh score of 5 group (16/30, $53.3 \%)$ was higher than that with a Child-Pugh score of $6(10 / 23,43.5 \%)$. Likewise, ORR in the ALBI grade 1 group $(14 / 22,58.8 \%)$ was higher than that in the ALBI grade 2 group (12/31; 38.7\%; Table 2). The mTTP of the 53 patients was 8.5 months (95\% CI: 6.9-13.8 months; Supplementary Figure 1). The TTP in the first-line group was significantly longer than that in the second-line group $(p<0.05$; Figure 1A). The TTP in the first-line group was significantly longer than that in the third-line group $(p<$ 0.01). The TTP in the BCLC stage B group tended to be longer than that in the stage $\mathrm{C}$ group $(p=0.07$; Figure 1B). Similarly, TTP in the ALBI Grade1 group was significantly longer than that in the ALBI Grade2 group $(p<0.05$; Figure 1C). Further, TTP in cases with a ChildPugh score of 5 was significantly longer than that in cases with a Child-Pugh score of 6 ( $p<0.01$; Figure 1D).

The median OS of the 53 patients was NA $(95 \%$ CI:19.8-NA months; Supplementary Figure 2). The median OS in the first-, second-, and third-line groups were not reached. The OS in the first-line group was significantly longer than that in the third-line group $(p<$ 0.05 ; Figure $2 \mathrm{~A}$ ). There was no significant difference in OS between the first- and second-line groups. The OS in the BCLC stage B group was significantly longer than that in the stage $\mathrm{C}$ group $(p<0.01$; Figure $2 \mathrm{~B})$. The $\mathrm{OS}$ in the ALBI Grade1 group tended to be longer than that in the ALBI Grade2 group ( $p<0.05$; Figure 2C). Moreover, OS with a Child-Pugh score of 5 was significantly longer than that with a score of 6 ( $p<0.05$; Figure 2D).

\section{Safety}

Grade 4 adverse effects (AEs) were not observed during the observation period. The most common allgrade drug-related AEs were hypertension $(54.7 \%$; $29 / 53)$, proteinuria $(47.2 \%$; $25 / 53)$, fatigue $(49.1 \%$; 
$26 / 53)$, appetite loss $(37.7 \% ; 20 / 53)$, and palmar-plantar erythrodysesthesia $(26.4 \% ; 14 / 53$; Table 3$)$. The most common grade 3 drug-related AEs were proteinuria $(24.5 \%, 13 / 53)$, hypertension $(15.1 \%, 8 / 53)$, fatigue $(7.5 \%, 4 / 53)$, and diarrhea $(3.8 \%, 2 / 53)$. There were no significant differences in LEN-related AEs among each treatment group. Moreover, the frequencies of LENrelated AEs were higher in the ALBI Grade2 group than in the ALBI Grade1 group (Table 4). Among them, the frequency of fatigue was significantly higher in patients in the ALBI-2 group $(23 / 31,74.2 \%)$ than in those in the ALBI-1 group (3/22 13.6\%; $p<0.01)$. Similar AE results were observed between groups comprising ChildPugh scores of 5 and 6 (data not shown). Treatment with
LEN was discontinued due to AEs in only three patients. All AEs were controlled by appropriate dose reduction or care.

\section{Drug administration}

The relative dose intensity (RDI) in the first-, second-, and third-line groups was $83.1 \%, 73.6 \%$, and $73.5 \%$, respectively. Treatment continued for 12 weeks in all cases, except in one case of PD and three cases of withdrawal due to AEs (two cases of grade 3 fatigue). During 12 weeks of observation, AEs led to the interruption of LEN administration in eight $(15.0 \%)$ patients and dose reduction in $13(24.5 \%)$.
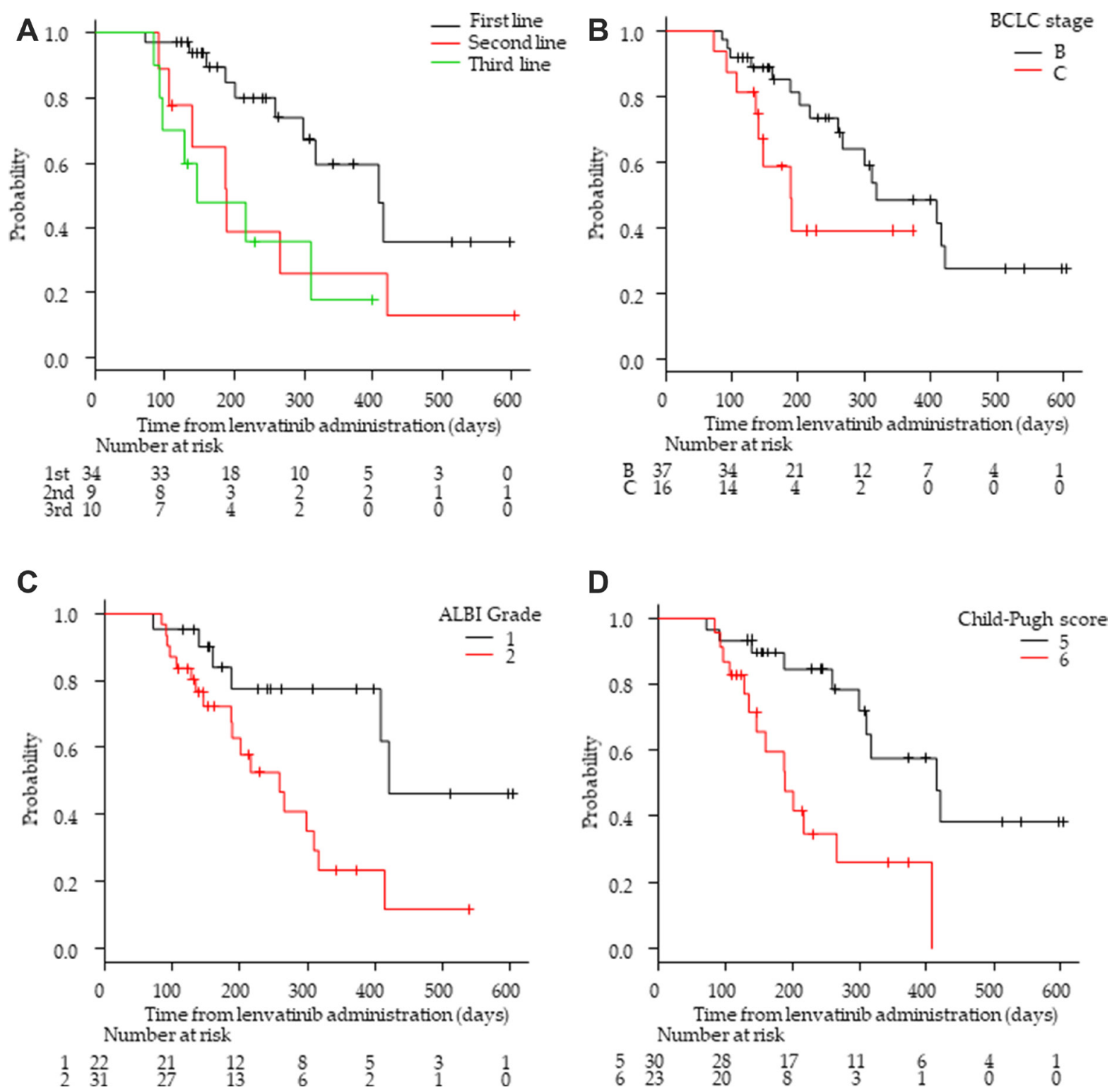

Figure 1: Kaplan-Meier analysis of progression-free survival among patients with advanced hepatocellular carcinoma treated with lenvatinib according to treatment lines and hepatic functional reserve. (A) First/second/third-line groups. (B) Barcelona Clinic Liver Cancer (BCLC) stage B and C groups. (C) Albumin-bilirubin (ALBI) grade 1 and 2 group (D) Child-Pugh score 5 and 6 groups. 
Table 2: Response to treatment with lenvatinib for advanced hepatocellular carcinoma according to treatment line, stage, and hepatic functional reserve

\begin{tabular}{|c|c|c|c|c|c|c|}
\hline Evaluation (mRECIST) & CR & PR & SD & PD & ORR (\%) & DCR (\%) \\
\hline \multicolumn{7}{|l|}{ Treatment line } \\
\hline First-line $(n=34)$ & $1(2.9)$ & $20(58.8)$ & $12(35.3)$ & $1(2.9)$ & 61.8 & 97.1 \\
\hline Second-line $(n=9)$ & $1(11.1)$ & $2(22.2)$ & $5(55.5)$ & $1(14.3)$ & 33.3 & 88.8 \\
\hline Third-line $(n=10)$ & $0(0)$ & $2(20.0)$ & $8(80.0)$ & $0(0)$ & 20.0 & 100 \\
\hline \multicolumn{7}{|l|}{ BCLC stage } \\
\hline $\mathrm{B}(n=37)$ & $2(5.4)$ & $18(48.6)$ & $17(45.9)$ & $0(0)$ & 54.1 & 100 \\
\hline $\mathrm{C}(n=16)$ & $0(0)$ & $6(37.5)$ & $8(50.0)$ & $2(12.5)$ & 37.5 & 87.5 \\
\hline \multicolumn{7}{|l|}{ Child-Pugh score } \\
\hline $5(n=30)$ & $2(6.7)$ & $14(46.7)$ & $12(40.0)$ & $2(6.7)$ & 53.3 & 93.3 \\
\hline $6(n=23)$ & $0(0)$ & $10(43.5)$ & $13(56.5)$ & $0(0)$ & 43.5 & 100 \\
\hline \multicolumn{7}{|l|}{ ALBI grade } \\
\hline $1(n=22)$ & $1(4.5)$ & $13(59.0)$ & $6(27.2)$ & $2(9.1)$ & 63.6 & 91.0 \\
\hline $2(n=31)$ & $1(3.2)$ & $11(35.5)$ & $19(61.3)$ & $0(0)$ & 38.7 & 100 \\
\hline
\end{tabular}

Abbreviations: mRECIST, modified response evaluation criteria in solid tumors; BCLC, Barcelona Clinic Liver Cancer; ALBI, albumin-bilirubin; CR, complete response; PR, partial response; SD, stable disease; PD, progressive disease; ORR, overall response rate; DCR, disease control rate.

\section{In vitro viability of the SOR-resistant cell line after LEN treatment}

To confirm our clinical observations and to analyze mechanisms underlying the sensitivity of $\mathrm{HCC}$ cells to LEN in vitro, we first performed a cell viability assay using previously established PLC/PRF5 and SOR-resistant PLC/PRF5-R2 cell lines [20]. The $\mathrm{IC}_{50}$ value of LEN towards PLC/PRF5 cells was $6.4 \mu \mathrm{M}$, and this value was consistent with those of previous reports [20]. However, for SOR-resistant PLC/PRF5-R2 cells, the $\mathrm{IC}_{50}$ value of LEN was $30 \mu \mathrm{M}$, which was significantly higher than that with the parental PLC/PRF5 cells ( $p<0.05$; Figure 3A). These findings suggested that PLC/PRF5-R2 cells might show partial cross-resistance to LEN.

\section{In vitro signal transduction pathway analysis in SOR-resistant cell lines}

LEN reportedly inhibits the phosphorylation of tyrosine-kinases such as FGF receptors, VEGF receptors, and the PDGF receptors RET and KIT [12-15]. Therefore, we investigated the degree of protein phosphorylation related to LEN-related signal transduction pathways in response to LEN using a comprehensive protein phosphorylation array (Supplementary Figure 3). A representative heatmap of array results including cluster analysis is shown in Supplementary Figure 4. The heatmap demonstrated that protein phosphorylation levels in all 377 signaling pathways were clearly distinguished and clustered between PLC/PRF5 and PLC/PRF5-R2 cells following treatment with LEN. The number of LEN-related signal transduction pathways that were significantly altered following LEN treatment was 16 including 12 related to FGFR, three to PDGF, and one to
VEGF, whereas only three FGFR pathways were altered in PLC/PRF5-R2 cells (Figure 3B). These results further indicated that PLC/PRF5-R2 cells show cross-resistance to LEN.

\section{In vitro FRS2-related pathway analysis in the SOR-resistant cell line}

The adaptor protein fibroblast growth factor receptor substrate 2 (FRS2) is reportedly an essential downstream component of the FGFR signaling pathway and acts as a hub linking several signaling pathways to ultimately activate FGFRs [21]. Therefore, we examined the inhibitory effects of LEN on the phosphorylation of 63 FRS2-related pathways among a total of 377 pathways involved in signal transduction (Supplementary Table 2) in PLC/PRF5 and PLC/PRF5-R2 cell lines using a phosphorylation array (Figure 3C). LEN suppressed the phosphorylation of FRS2 protein in those pathways in both cell lines. However, the degree of suppression was significantly higher in PLC/PRF5 cells than in PLC/ PRF5-R2 cells $(p<0.01$, Welch's $t$-test); this indicated that the degree of LEN-mediated inhibition of FRS2related signaling pathways in PLC/PRF5-R2 cells was significantly lower than that in PLC/PRF5 cells. Thus, it was evident that SOR-resistant HCC cells show partial cross-resistance to LEN based on resistance to the LENmediated inhibition of FGFR signaling pathways.

\section{DISCUSSION}

In this retrospective study, we demonstrated the therapeutic efficacy and safety of LEN as a second- and third-line treatment, particularly for patients intolerant to SOR (second-line) and as a first-line treatment for HCC. 
Moreover, our results suggested that treatment with LEN, while maintaining better hepatic functional reserves, might exert more beneficial effects on the prognosis of patients with advanced HCC in a clinical setting. Furthermore, our in vitro experiments revealed that the SOR-resistant cell line PLC/PRF5-R2 was partially crossresistant to LEN. LEN significantly inhibited 16 signal transduction pathways including 12 FGFR pathways in parent PLC/PRF5 cells but inhibited only a few pathways in PLC/PRF5-R2 cells. These results support our clinical observations indicating that the response rate of third-line LEN treatment was rather low compared to that of firstline treatments.

At present, REG, CAB, and RAM have resulted in prolonged OS compared to that with placebo for patients with SOR-resistant disease [4, 10, 22]. However, there has been no clear indication to use these drugs as a second- line treatment since there is no head-to-head comparison available for drugs in this setting. REG has been used only to treat patients who are tolerant to SOR treatment, as a second-line treatment following SOR, but not for patients intolerant to SOR. Approximately one third of patients who receive SOR treatment are reportedly intolerant to SOR [5-8], and therefore are deemed unsuitable to receive substantially effective systemic chemotherapy. Thus, LEN shows potential as a second-line treatment for patients with unresectable HCC intolerant to SOR.

In this study, the ORRs of LEN in third-line treatment $(20.0 \%)$ were significantly lower than those in first-line treatment group (61.8\%) and somewhat lower than those in the second-line treatment group (33.3\%). However, DCR in the third-line treatment group was highly similar to that with the other-line treatments. Likewise, the mTTP and OS in the third-line treatment
A

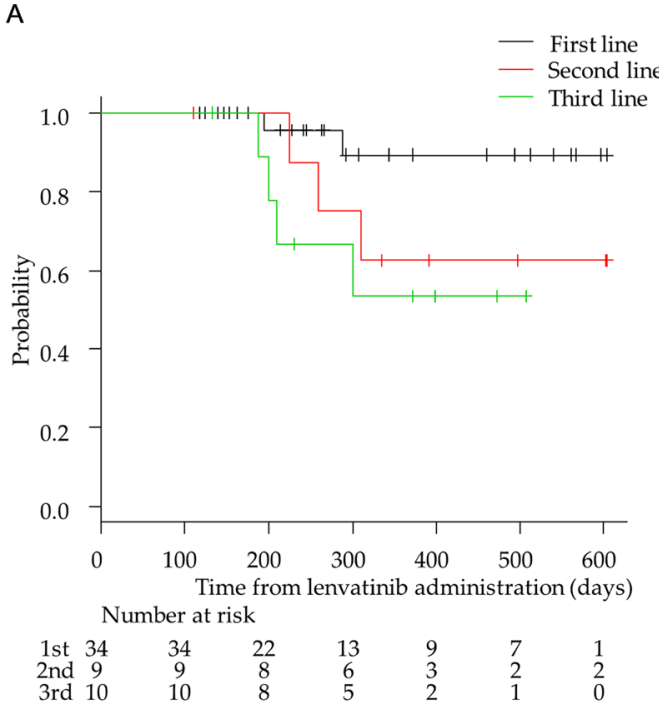

C



B
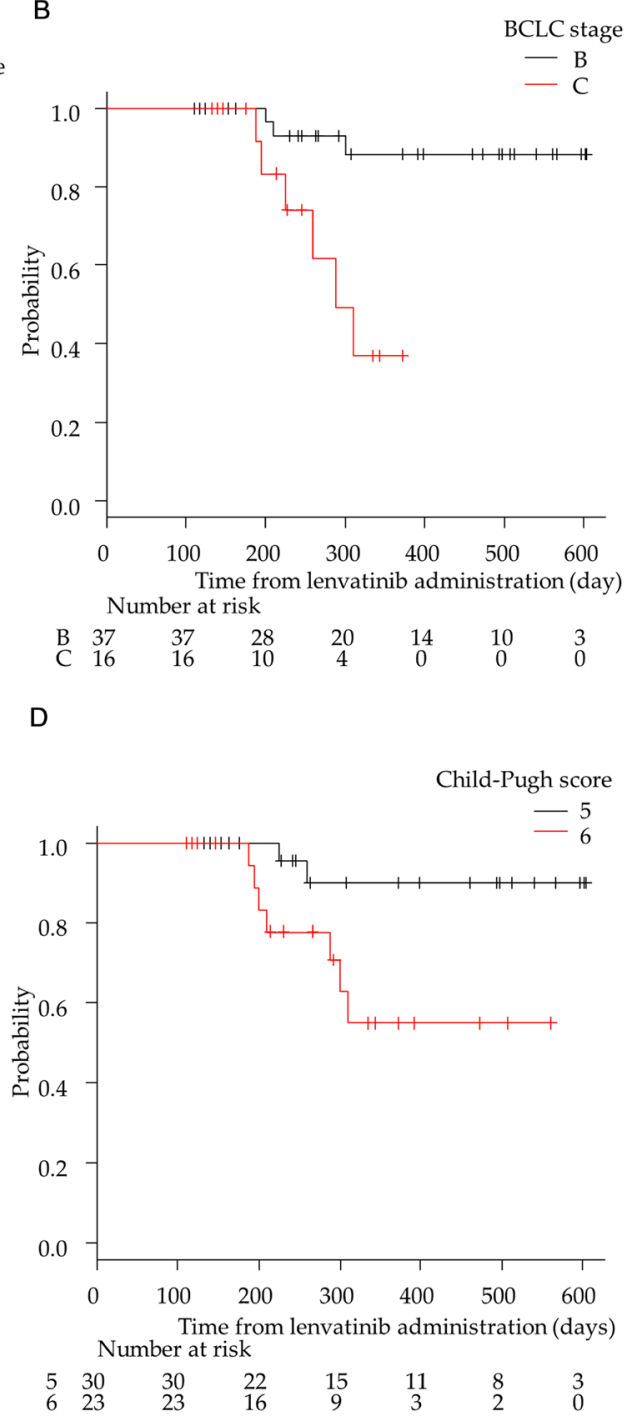

Figure 2: Kaplan-Meier analysis of overall survival among patients with advanced hepatocellular carcinoma treated with lenvatinib according to treatment lines and hepatic functional reserve. (A) First-/second-/third-line groups. (B) Barcelona Clinic Liver Cancer (BCLC) stage B and C groups. (C) Albumin-bilirubin (ALBI) grade 1 and 2 group (D) Child-Pugh score 5 and 6 groups. 
Table 3: Adverse events associated with lenvatinib treatment

\begin{tabular}{|c|c|c|c|c|c|c|c|c|c|c|}
\hline \multirow[t]{2}{*}{ Event } & \multicolumn{2}{|c|}{ All $(n=53)$} & \multicolumn{2}{|c|}{ First-line $(n=34)$} & \multicolumn{2}{|c|}{ Second-line $(n=9)$} & \multicolumn{2}{|c|}{ Third-line $(n=10)$} & \multicolumn{2}{|c|}{$p$-value } \\
\hline & $\begin{array}{l}\text { Any } \\
\text { Grade }\end{array}$ & Grade3 & $\begin{array}{l}\text { Any } \\
\text { Grade }\end{array}$ & Grade3 & $\begin{array}{l}\text { Any } \\
\text { Grade }\end{array}$ & Grade3 & $\begin{array}{l}\text { Any } \\
\text { Grade }\end{array}$ & Grade3 & $\begin{array}{c}\text { Any } \\
\text { Grade }\end{array}$ & Grade3 \\
\hline Hypertension & $29(54.7)$ & $8(15.1)$ & $21(61.8)$ & $7(20.6)$ & $3(33.3)$ & $1(11.1)$ & $5(50.0)$ & $0(0)$ & 0.32 & 0.35 \\
\hline Fatigue & $26(49.1)$ & $4(7.5)$ & $16(47.1)$ & $1(2.9)$ & $3(33.3)$ & $1(11.1)$ & $7(70.0)$ & $2(20.0)$ & 0.27 & 0.13 \\
\hline Decreased appetite & $20(37.7)$ & $0(0)$ & $13(36.0)$ & $0(0)$ & $2(22.2)$ & $0(0)$ & $5(50.0)$ & $0(0)$ & 0.55 & - \\
\hline Decreased platelet count & $12(22.6)$ & $0(0)$ & $9(38.2)$ & $0(0)$ & $2(22.2)$ & $0(0)$ & $1(10.0)$ & $0(0)$ & 0.57 & - \\
\hline Palmar-plantar erythrodysesthesia & $14(26.4)$ & $0(0)$ & $7(20.6)$ & $0(0)$ & $3(33.3)$ & $0(0)$ & $4(40.0)$ & $0(0)$ & 0.32 & - \\
\hline Proteinuria & $25(47.2)$ & $13(24.5)$ & $16(47.1)$ & $10(29.4)$ & $3(33.3)$ & $1(11.1)$ & $6(60.0)$ & $2(20.0)$ & 0.16 & 0.59 \\
\hline Diarrhea & $10(18.9)$ & $2(3.8)$ & $4(11.8)$ & $2(5.9)$ & $3(33.3)$ & $0(0)$ & $3(30.0)$ & $0(0)$ & 0.26 & 1 \\
\hline Increased blood bilirubin & $3(5.7)$ & $0(0)$ & $1(2.9)$ & $0(0)$ & $0(0)$ & $0(0)$ & $2(20.0)$ & $0(0)$ & 0.16 & - \\
\hline Dysphonia & $8(15.1)$ & $0(0)$ & $5(14.7)$ & $0(0)$ & $3(33.3)$ & $0(0)$ & $0(0)$ & $0(0)$ & 0.13 & - \\
\hline $\begin{array}{l}\text { Elevated-aspartate } \\
\text { aminotransferase }\end{array}$ & $4(7.5)$ & $0(0)$ & $2(5.9)$ & $0(0)$ & $1(11.1)$ & $0(0)$ & $1(10.0)$ & $0(0)$ & 0.61 & - \\
\hline Hypothyroidism & $6(11.3)$ & $0(0)$ & $4(11.8)$ & $0(0)$ & $2(22.2)$ & $0(0)$ & $1(10.0)$ & $0(0)$ & 0.72 & - \\
\hline Hepatic coma & $1(1.9)$ & - & $0(0)$ & - & $1(11.1)$ & - & $0(0)$ & - & 0.36 & - \\
\hline
\end{tabular}

The $p$-values were calculated with Fisher's exact test.

group were also significantly shorter than those in the first-line treatment group and tended to be shorter than those in the second-line treatment groups.

To date, there have been no studies investigating ORRs for second- and third-line treatment regimens using LEN. Hiraoka et al. reported that there were no significant differences in the efficacy of LEN for advanced HCC between MTA-naïve and MTA-experienced groups [18]. Although ORRs for each patient group were not shown, the MTA-experienced patients included only $25 \%(11 / 44)$ of patients following SOR-REG treatment (third line), whereas $75 \%$ (33/44) were considered SOR-intolerant patients (second line). In this context, it appears that LEN is relatively effective for SOR-intolerant patients but less effective for patients resistant to SOR-REG treatment. In fact, the effects of LEN in our second-line cohort were comparable to those with first-line treatment. This might be due to the fact that patients administered second-line LEN treatment could still be sensitive to MTAs since they could not continue SOR treatment due to detrimental adverse effects but did not acquire complete resistance to SOR. Therefore, it is reasonable to assume that LEN is fairly effective for HCC patients intolerant to SOR as a second-line treatment.

Our in vitro cell viability assay revealed that the $\mathrm{IC}_{50}$ of LEN towards the SOR-resistant cell line (PLC/ PRF5-R2) was significantly higher than that with parental PLC/PRF5 cells ( $p<0.01$; Figure 3A). It is plausible that the signaling pathway inhibited by LEN in HCC cells was modified during the course of acquiring SORresistance, leading to partial cross-resistance to LEN. In general, it is difficult to delineate the multifaceted and dynamic pathway regulation in response to MTAs such as LEN. Therefore, we employed a comprehensive protein phosphorylation array, which can simultaneously measure the phosphorylation degrees of 1205 proteins belonging to 377 signal transduction pathways (Supplementary Figure 3) [23]. As a result, we confirmed that LEN mainly inhibited the phosphorylation of 12 FGFR-related pathways in parental PLC/PRF5 cells, consistent with previous reports showing that LEN selectively suppresses the proliferation of HCC cells with activated FGF signaling pathways; this is a distinct feature of LEN as compared to that with SOR [21]. Conversely, only three FGFR-related signaling pathways were significantly inhibited in PLC/PRF5-R2 cells, indicating the partial resistance of those SOR-resistant cells to LEN. These data were further supported by the fact that the phosphorylation degree of FRS2, which plays a pivotal role in FGFRrelated pathways, in PLC/PRF5-R2 cells was significantly higher than that in parental PLC/PRF5 cells (Figure 3C). Thus, our protein array analysis suggests that LEN is less effective for HCC patients with resistance to SOR than for SOR-naïve patients due to cross-resistance between LEN and SOR.

The AE profiles in this study were similar to those in previous reports [18, 19, 24], mostly documented during first-line treatment. Otherwise, the incidence of AEs with second/third line treatment was similar to that with firstline treatment (Table 3). This might be explained by the particular characteristics of LEN, which shows different $\mathrm{AE}$ spectra from those of both SOR and REG. Moreover, treatment with LEN could easily be initiated as a second/ third-line treatment following treatment with SOR or REG, even in patients suffering from severe AEs related to SOR or REG such as hand-foot syndrome and diarrhea. Thus, our data demonstrated the safety and feasibility of LEN as a second/third-line treatment for unresectable HCC. In addition, the incidence of fatigue in the ALBI2 group containing all treatment lines was significantly higher than that in the ALBI-1 group (Table 4). This fatigue was often the cause of dose reduction and the 
A

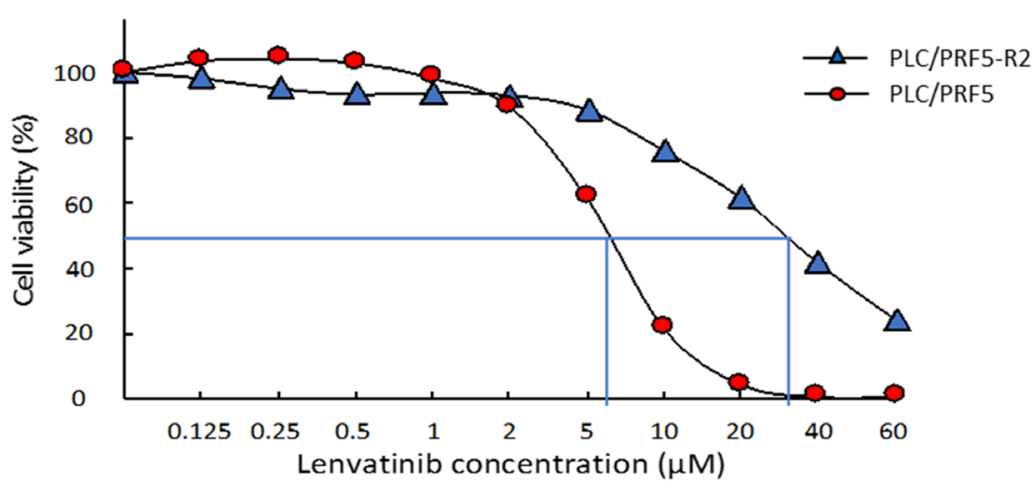

B

\begin{tabular}{|c|c|c|c|}
\hline \multicolumn{2}{|l|}{ PLC/PRF5_LEN } & \multicolumn{2}{|c|}{ PLC/PRF5-R2_LEN } \\
\hline Pathway & Pathway category & Pathway & Pathway category \\
\hline Negative_regulation_of_FGFR3_signaling_B & Signaling_by_FGFR & Negative_regulation_of_FGFR3_signaling_B & Signaling_by_FGFR \\
\hline Spry_regulation_of_FGF & Signaling_by_FGFR & Spry_regulation_of_FGF & Signaling_by_FGFR \\
\hline p-CBL_GRB2_binds_p-FRS2_activated_FGFR1 & Signaling_by_FGFR & Negative_regulation_of_FGFR2 & Signaling_by_FGFR \\
\hline Negative_regulation_of_FGFR1 & Signaling_by_FGFR & & \\
\hline Activated_FGFR1_p-FRS2_p-PPTN11_binds_GRB2_GAB1_PI3KR1 & Signaling_by_FGFR & & \\
\hline Activated_FGFR1_p-FRS2_p-PPTN11_GRB2_GAB1_PIK3R1_binds_PIK3CA & Signaling_by_FGFR & & \\
\hline Activated_FGFR3_p-FRS2_p-PPTN11_GRB2_GAB1_PIK3R1_binds_PIK3CA & Signaling_by_FGFR & & \\
\hline CBL_ubiquitinates_FRS2_and_FGFR1 & Signaling_by_FGFR & & \\
\hline FRS-mediated_FGFR1_signaling & Signaling_by_FGFR & & \\
\hline CBL_ubiquitinates_FRS2_and_FGFR3 & Signaling_by_FGFR & & \\
\hline Activated_FGFR1_p-FRS_bind_to_PPTN11 & Signaling_by_FGFR & & \\
\hline Activated_FGFR1_p-SHC1_binds_GRB2_SOS1 & Signaling_by_FGFR & & \\
\hline STAT_binds_to_the_active_receptor & Signaling_by_PDGF & & \\
\hline SHP2_binds_to_the_active_receptor & Signaling_by_PDGF & & \\
\hline Grb2-Sos1_complex_binds_to_the_active_receptor & Signaling_by_PDGF & & \\
\hline p38_MAPK_activation_by_VEGFR & Signaling_by_VEGF & & \\
\hline
\end{tabular}

C

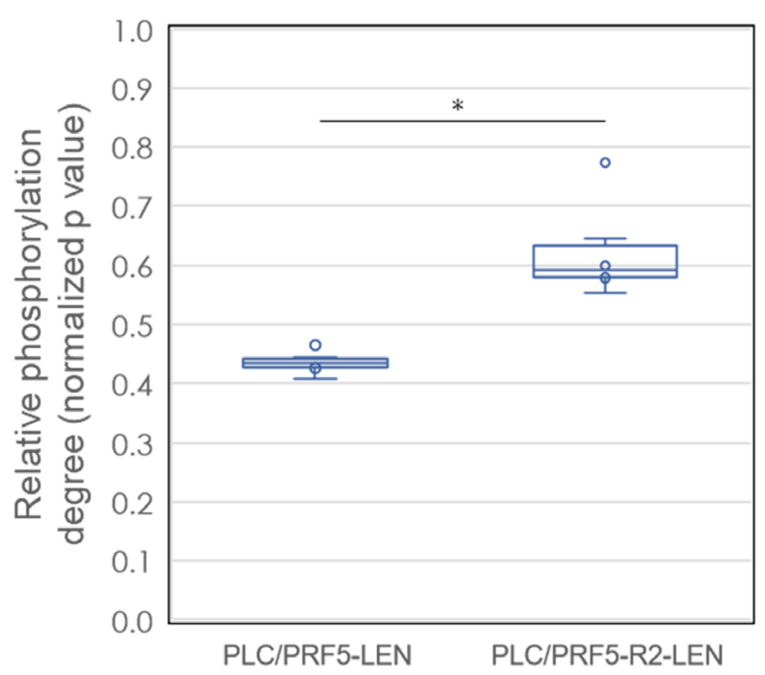

Figure 3: In vitro cell viability and signal transduction pathway analysis of sorafenib (SOR)-resistant cell line with lenvatinib treatment by a comprehensive protein phosphorylation array. (A) The sensitivities of PLC/PRF5 and PLC/ PRF5-R2 to lenvatinib (LEN) were assessed by MTT assays. (B) A list of LEN-related signal transduction pathways significantly altered after treatment with LEN in PLC/PRF5 and PLC/PRF5-R2 cells. The 114 LEN related pathways were categorized into "Signaling by FGFR", "Signaling by PDGF", "Signaling by VEGFR", and "Signaling by SRC-KIT" among 377 pathways tested. (C) Boxplot analysis of degree of phosphorylation of associated proteins belonging to the 63 FRS2-related transduction pathways in PLC/PRF5 and PLC/ PRF5-R2 cells in response to LEN. The boxes show the interquartile rage with the median value indicated by the horizontal line; whiskers show the range and circles indicate outliers. ${ }^{*} p<0.0014$. 
Table 4: The relationship between adverse events and ALBI-grade

\begin{tabular}{lccc}
\hline \multirow{2}{*}{ Event } & \multicolumn{3}{c}{ ALBI grade $\boldsymbol{n}=\mathbf{5 3}$} \\
\cline { 2 - 4 } Hypertension & ALBI-1 $\boldsymbol{n}=\mathbf{2 2}$ & ALBI-2 $\boldsymbol{n}=\mathbf{3 1}$ & $\boldsymbol{p}$-value \\
Fatigue & $11(50.0)$ & $18(58.1)$ & 0.59 \\
Decreased appetite & $3(13.6)$ & $23(74.2)$ & $<0.01$ \\
Decreased platelet count & $7(31.8)$ & $13(41.9)$ & 0.57 \\
Palmar-plantar erythrodysesthesia & $4(18.2)$ & $8(25.8)$ & 0.74 \\
Proteinuria & $5(22.7)$ & $9(29.0)$ & 0.76 \\
\hline
\end{tabular}

The $p$-values were calculated with Fisher's exact test. Abbreviations: ALBI, albumin-bilirubin.

interruption of treatment, especially in the ALBI-2 group. Since the ALBI score is calculated from only albumin and total bilirubin values, the ALBI-2 group is more likely to have lower serum albumin levels, reflecting poorer nutrition and performance status, which might more readily lead to fatigue in HCC patients [25-27].

In this study, patients with better liver functional reserves (Child-Pugh score of 5, ALBI Grade1) showed better response to LEN and longer OS. Recently, Ueshima and associates analyzed 82 patients with unresectable HCCs treated with LEN and reported that ALBI Grade1 and serum AFP levels $<200$ are predictors of a high response rate [28]. They also demonstrated that the time to treatment failure in patients with better liver functional reserves was longer. These patients included 61.0\% MTAnaïve, $24.4 \%$ second-line (SOR intolerance), and only $14.6 \%$ third-line treatment patients. They also included those with a Child-Pugh score B, as well as those with score A, and BCLC stages A, B, and C. Although the specific proportions of patients were largely different from those in our study, our data on treatment outcomes displayed partial similarity to theirs. Patients with better liver functional reserves showed better responses, and the OS in the Child-Pugh score 5 group was significantly longer than that in the Child-Pugh score 6 group. These results might be partly explained by the difference in RDI. The RDI of LEN in the Child-Pugh score 5 group $(81.4 \%)$ was higher than that in the Child-Pugh score 6 group $(76.5 \%)$. Thus, to maximize the therapeutic effect of LEN, it should be used in patients with unresectable HCC, while liver function is preserved, as with Child-Pugh A and ALBI-1 grade patients. Although repeated TACE was often performed for the treatment of unresectable HCC until recently, LEN treatment should be initiated in HCC patients with better liver functional reserves. Eventually, in this study, patients with BCLC stage $\mathrm{C}$ showed lower ORRs and a shorter TTP and OS than those with BCLC stage $\mathrm{B}$. One of the reasons is that BCLC stage $\mathrm{C}$ cases included six patients with portal vein invasion, which might be related to the poor outcomes observed.

One limitation of this study was its single-center, retrospective design. Of note, the sex distribution of the research population was uneven in this cohort. This might be due to the fact that the male sex correlates with the development of HCC among patients with cirrhosisrelated HCV [29], which was observed at a relatively high rate in our cohort.

Another limitation was that the observation period was relatively short and the number of analyzed patients was small. However, considering that LEN had only been approved in Japan for 25 months, our observations at the specified cutoff date are adequate to report real-world treatment results, especially those related to evaluating the initial safety and efficacy of the clinical use of LEN. A large-scale prospective study is indispensable to establish the efficacy of LEN for second-and third line-treatment use in the future. Regarding in vitro experiments, LEN exerts its effect by blocking not only FGFR, but also PDGFR- $\alpha$ and VEGFR, the latter of which is expressed in endothelial cells rather than cancer cells. Therefore, the findings should be confirmed using in vivo HCC xenograft models, where the anti-angiogenic activity of LEN can be evaluated.

In conclusion, we demonstrated that LEN monotherapy could be feasible as a second/third-line treatment for unresectable HCC and suggest that LEN should preferably be applied to patients with better functional liver reserves (ALBI-1 or Child score 5) to obtain good outcomes. Moreover, LEN was more effective in MTA-naïve patients as a first-line treatment than in patients administered LEN for second- and thirdline treatment, and particularly in patients on third-line treatment after a SOR-REG treatment. These clinical data are supported by the in vitro experimental results indicating that the SOR-resistant cell line became partially cross-resistant to LEN by altering FGFR-related signal transduction pathways.

\section{MATERIALS AND METHODS}

\section{Patient selection and diagnosis of $\mathrm{HCC}$}

This retrospective, observational study evaluated the efficacy and safety of LEN (Lenvima ${ }^{\circledR}$, Eisai Co., Ltd., Tokyo, Japan) monotherapy in patients with unresectable advanced HCC at Tokushima University Hospital between March and December 2018. This study was approved by the Ethics Committee of Tokushima University Hospital 
(Approval number; 3489). The inclusion criteria were based on those of the REFLECT trial. Briefly, eligible patients had target lesions defined as measurable based on mRECIST [30], an ECOG PS score of 0 or 1 [31], BCLC $\mathrm{B}$ or $\mathrm{C}$ categorizations [32], and Child-Pugh class A. Written informed consent was obtained from all patients. The diagnosis of HCC was based on guidelines established by the Liver Cancer Study Group of Japan [33]. According to these guidelines, a diagnosis of $\mathrm{HCC}$ was confirmed via histology or characteristic radiologic findings such as typical arterial enhancement of the tumor followed by a washout pattern in the images of the portal venous phase or the equilibrium phase obtained by dynamic spiral CT imaging or contrast-enhanced MRI.

\section{Treatment with LEN}

The initial daily oral doses of LEN given to patients weighing $\geq 60,<60$, and $<40 \mathrm{~kg}$ were 12,8 , and $4 \mathrm{mg} /$ day, respectively. The initial daily oral doses of LEN given to patients weighing $\geq 60$ and $<60 \mathrm{~kg}$ were 12 and $8 \mathrm{mg}$ /day, respectively. For HCC patients weighing $<40$ $\mathrm{kg}$, we started with the initial LEN dose of $4 \mathrm{mg} /$ day and confirmed the safety for 1 week, which was followed by dosing up to $8 \mathrm{mg}$ /day since there are no reports showing the appropriate starting dose for patients weighing $<40 \mathrm{~kg}$.

When serious AEs were observed, LEN administration was discontinued. Dose interruptions were in accordance with medical package inserts for administering LEN. Briefly, when Grade3 AEs or unacceptable Grade2 AEs developed, LEN was discontinued until AEs recovered and reverted to a lower grade.

\section{Hepatic reserve function}

Hepatic reserve function was assessed according to ALBI grading and Child-Pugh classification. ALBI grade was calculated based on serum albumin and total bilirubin values using the following formula: $\left[\right.$ ALBI score $=\left(\log _{10}\right.$ bilirubin $(\mu \mathrm{mol} / \mathrm{L}) \times 0.66)+($ albumin $(\mathrm{g} / \mathrm{L}) \times-0.085)]$ and defined by the following scores: $\leq-2.60=$ Grade $1,>$ -2.60 to $\leq-1.39=$ Grade $2,>-1.39=$ Grade 3 [34].

\section{Follow-up and patient outcome}

Patients were observed for at least 12 weeks. Safety was assessed by recording any adverse drug reactions, clinical laboratory tests, physical examination, measurement of vital signs, hematological and biochemical laboratory testing, and urinalysis. Adverse drug reactions were defined according to the Common Terminology Criteria for Adverse Events version 5.0. Radiologic; responses to therapy were evaluated according to mRECIST at the 8th week after starting LEN and every 8 weeks thereafter. ORR was defined as the sum of CR and $\mathrm{PR}$ rates. DCR was defined as the sum of $\mathrm{CR}, \mathrm{PR}$, and SD rates. TTP was defined as the time from the first day of administering LEN until the day of radiological progression.

\section{Cell culture and viability analysis}

The representative human hepatoma cell line PLC/ PRF5 was purchased from the American Tissue Culture Collection (ATCC, Manassas, VA, USA). Establishment of the SOR-resistant PLC/PRF5 cell line (PLC/PRF5-R2) was performed as described previously [20]. Cells were grown in Dulbecco's modified Eagle's medium (Invitrogen Sigma-Aldrich, St. Louis, MO, USA) supplemented with $10 \% \mathrm{FBS}$ and $2 \mathrm{mM}$ L-glutamine. Cell viability was assessed via a 3-(4, 5-dimethylthiazol-2-yl)-2,5-diphenyl2H-tetrazolium bromide (MTT) assays as described previously [20].

\section{Identification of active signal transduction pathways by protein array}

We used a self-made comprehensive protein phosphorylation array that included 1205 proteins representing 377 pathways involved in signal transduction [23], as described in the supporting information (Supplementary Figure 3) to determine the phosphorylation status of selected proteins in the active signal transduction pathways of HCC cells in the absence or presence of LEN.

\section{Statistical analysis}

Categorical variables were compared using the Fischer's exact test, whereas continuous variables were compared using Mann-Whitney and Kruskal-Wallis tests. All significance tests were two-tailed, and statistical significance was set at $p<0.05$. Kaplan-Meier plots of medians (with 95\% CIs) were used to estimate TTP. All statistical analyses were undertaken using Easy R (EZR) version 1.29 (Saitama Medical Center, Jichi Medical University, Saitama, Japan).

\section{Author contributions}

All authors contributed to the study conception and design. Material preparation, data collection, and analysis were performed by Tetsu Tomonari, Hironori Tanaka, Takahiro Tanaka, Yasuteru Fujino, Yasuhiro Mitsui, Koichi Okamoto, Hiroshi Miyamoto, Naoki Muguruma, Harumi Kagiwada, Masashi Kitazawa, Kazuhiko Fukui, and Katsuhisa Horimoto. The first draft of the manuscript was written by Tetsu Tomonari, Yasushi Sato, and Tetsuji Takayama, and all authors commented on previous versions of the manuscript. All authors read and approved the final manuscript. 


\section{CONFLICTS OF INTEREST}

The authors declare they have no conflicts of interest.

\section{REFERENCES}

1. Siegel RL, Miller KD, Jemal A. Cancer statistics, 2018. CA Cancer J Clin. 2018; 68:7-30. https://doi.org/10.3322/ caac. 21442. [PubMed]

2. Llovet JM, Ricci S, Mazzaferro V, Hilgard P, Gane E, Blanc JF, de Oliveira AC, Santoro A, Raoul JL, Forner A, Schwartz M, Porta C, Zeuzem S, et al, and SHARP Investigators Study Group. Sorafenib in advanced hepatocellular carcinoma. N Engl J Med. 2008; 359:378390. https://doi.org/10.1056/NEJMoa0708857. [PubMed]

3. Cheng AL, Kang YK, Chen Z, Tsao CJ, Qin S, Kim JS, Luo R, Feng J, Ye S, Yang TS, Xu J, Sun Y, Liang H, et al. Efficacy and safety of sorafenib in patients in the AsiaPacific region with advanced hepatocellular carcinoma: a phase III randomised, double-blind, placebo-controlled trial. Lancet Oncol. 2009; 10:25-34. https://doi.org/10.1016/ S1470-2045(08)70285-7. [PubMed]

4. Bruix J, Qin S, Merle P, Granito A, Huang YH, Bodoky G, Pracht M, Yokosuka O, Rosmorduc O, Breder V, Gerolami $\mathrm{R}$, Masi G, Ross PJ, et al, and RESORCE Investigators. Regorafenib for patients with hepatocellular carcinoma who progressed on sorafenib treatment (RESORCE): a randomised, double-blind, placebo-controlled, phase 3 trial. Lancet. 2017; 389:56-66. https://doi.org/10.1016/S01406736(16)32453-9. [PubMed]

5. Ogasawara S, Chiba T, Ooka Y, Suzuki E, Maeda $\mathrm{T}$, Yokoyama M, Wakamatsu $\mathrm{T}$, Inoue $\mathrm{M}$, Saito $\mathrm{T}$, Kobayashi K, Kiyono S, Nakamura M, Nakamoto S, et al. Characteristics of patients with sorafenib-treated advanced hepatocellular carcinoma eligible for second-line treatment. Invest New Drugs. 2018; 36:332-339. https://doi. org/10.1007/s10637-017-0507-3. [PubMed]

6. Uchikawa S, Kawaoka T, Aikata H, Kodama K, Nishida Y, Inagaki Y, Hatooka M, Morio K, Nakahara T, Murakami E, Hiramatsu A, Tsuge M, Imamura M, et al. Clinical outcomes of sorafenib treatment failure for advanced hepatocellular carcinoma and candidates for regorafenib treatment in realworld practice. Hepatol Res. 2018; 48:814-820. https://doi. org/10.1111/hepr.13180. [PubMed]

7. Kuzuya T, Ishigami M, Ishizu Y, Honda T, Hayashi K, Ishikawa T, Nakano I, Hirooka Y, Goto H. Prognostic factors associated with postprogression survival in advanced hepatocellular carcinoma patients treated with sorafenib not eligible for second-line regorafenib treatment. Oncology. 2018; 95:91-99. https://doi.org/10.1159/000488453. [PubMed]

8. Terashima T, Yamashita T, Sunagozaka H, Arai K, Kawaguchi K, Kitamura K, Yamashita T, Sakai Y, Mizukoshi E, Honda M, Kaneko S. Analysis of the liver functional reserve of patients with advanced hepatocellular carcinoma undergoing sorafenib treatment: Prospects for regorafenib therapy. Hepatol Res. 2018; 48:956-966. https://doi.org/10.1111/hepr.13196. [PubMed]

9. Kuzuya T, Ishigami M, Ito T, Ishizu Y, Honda T, Ishikawa T, Hirooka Y, Fujishiro M. Clinical characteristics and outcomes of candidates for second-line therapy, including regorafenib and ramucirumab, for advanced hepatocellular carcinoma after sorafenib treatment. Hepatol Res. 2019; 49:1054-1065. https://doi.org/10.1111/hepr.13358. [PubMed]

10. Abou-Alfa GK, Meyer T, Cheng AL, El-Khoueiry AB, Rimassa L, Ryoo BY, Cicin I, Merle P, Chen Y, Park JW, Blanc JF, Bolondi L, Klumpen HJ, et al. Cabozantinib in patients with advanced and progressing hepatocellular carcinoma. N Engl J Med. 2018; 379:54-63. https://doi. org/10.1056/NEJMoa1717002. [PubMed]

11. Kudo M, Finn RS, Qin S, Han KH, Ikeda K, Piscaglia F, Baron A, Park JW, Han G, Jassem J, Blanc JF, Vogel A, Komov D, et al. Lenvatinib versus sorafenib in first-line treatment of patients with unresectable hepatocellular carcinoma: a randomised phase 3 non-inferiority trial. Lancet. 2018; 391:1163-1173. https://doi.org/10.1016/S0140-6736(18)30207-1. [PubMed]

12. Matsui J, Funahashi Y, Uenaka T, Watanabe T, Tsuruoka A, Asada M. Multi-kinase inhibitor E7080 suppresses lymph node and lung metastases of human mammary breast tumor MDA-MB-231 via inhibition of vascular endothelial growth factor-receptor (VEGF-R) 2 and VEGF-R3 kinase. Clin Cancer Res. 2008; 14:5459-5465. https://doi. org/10.1158/1078-0432.CCR-07-5270. [PubMed]

13. Matsui J, Yamamoto Y, Funahashi Y, Tsuruoka A, Watanabe T, Wakabayashi T, Uenaka T, Asada M. E7080, a novel inhibitor that targets multiple kinases, has potent antitumor activities against stem cell factor producing human small cell lung cancer H146, based on angiogenesis inhibition. Int J Cancer. 2008; 122:664-671. https://doi.org/10.1002/ijc.23131. [PubMed]

14. Tohyama O, Matsui J, Kodama K, Hata-Sugi N, Kimura T, Okamoto K, Minoshima Y, Iwata M, Funahashi Y. Antitumor activity of lenvatinib (e7080): an angiogenesis inhibitor that targets multiple receptor tyrosine kinases in preclinical human thyroid cancer models. J Thyroid Res. 2014; 2014:638747. https://doi.org/10.1155/2014/638747. [PubMed]

15. Yamamoto Y, Matsui J, Matsushima T, Obaishi H, Miyazaki K, Nakamura K, Tohyama O, Semba T, Yamaguchi A, Hoshi SS, Mimura F, Haneda T, Fukuda Y, et al. Lenvatinib, an angiogenesis inhibitor targeting VEGFR/FGFR, shows broad antitumor activity in human tumor xenograft models associated with microvessel density and pericyte coverage. Vasc Cell. 2014; 6:18. https://doi.org/10.1186/2045824X-6-18. [PubMed]

16. Ikeda K, Kudo M, Kawazoe S, Osaki Y, Ikeda M, Okusaka T, Tamai T, Suzuki T, Hisai T, Hayato S, Okita K, Kumada H. Phase 2 study of lenvatinib in patients with advanced hepatocellular carcinoma. J Gastroenterol. 2017; 52:512519. https://doi.org/10.1007/s00535-016-1263-4. [PubMed] 
17. Kobayashi M, Kudo M, Izumi N, Kaneko S, Azuma M, Copher R, Meier G, Pan J, Ishii M, Ikeda S. Costeffectiveness analysis of lenvatinib treatment for patients with unresectable hepatocellular carcinoma (uHCC) compared with sorafenib in Japan. J Gastroenterol. 2019; 54:558-570. https://doi.org/10.1007/s00535-019-01554-0. [PubMed]

18. Hiraoka A, Kumada T, Kariyama K, Takaguchi K, Itobayashi E, Shimada N, Tajiri K, Tsuji K, Ishikawa T, Ochi H, Hirooka M, Tsutsui A, Shibata H, et al, and Reallife Practice Experts for HCC (RELPEC) Study Group and the HCC 48 Group (hepatocellular carcinoma experts from 48 clinics in Japan). Therapeutic potential of lenvatinib for unresectable hepatocellular carcinoma in clinical practice: Multicenter analysis. Hepatol Res. 2019; 49:111-117. https://doi.org/10.1111/hepr.13243. [PubMed]

19. Obi S, Sato T, Sato S, Kanda M, Tokudome Y, Kojima Y, Suzuki Y, Hosoda K, Kawai T, Kondo Y, Isomura Y, Ohyama H, Nakagomi K, et al. The efficacy and safety of lenvatinib for advanced hepatocellular carcinoma in a realworld setting. Hepatol Int. 2019; 13:199-204. https://doi. org/10.1007/s12072-019-09929-4. [PubMed]

20. Tomonari T, Takeishi S, Taniguchi T, Tanaka T, Tanaka H, Fujimoto S, Kimura T, Okamoto K, Miyamoto H, Muguruma N, Takayama T. MRP3 as a novel resistance factor for sorafenib in hepatocellular carcinoma. Oncotarget. 2016; 7:7207-7215. https://doi.org/10.18632/oncotarget.6889. [PubMed]

21. Wesche J, Haglund K, Haugsten EM. Fibroblast growth factors and their receptors in cancer. Biochem J. 2011; 437:199-213. https://doi.org/10.1042/BJ20101603. [PubMed]

22. Zhu AX, Kang YK, Yen CJ, Finn RS, Galle PR, Llovet JM, Assenat E, Brandi G, Pracht M, Lim HY, Rau KM, Motomura K, Ohno I, et al, and REACH-2 study investigators. Ramucirumab after sorafenib in patients with advanced hepatocellular carcinoma and increased alphafetoprotein concentrations (REACH-2): a randomised, double-blind, placebo-controlled, phase 3 trial. Lancet Oncol. 2019; 20:282-296. https://doi.org/10.1016/S14702045(18)30937-9. [PubMed]

23. Manabe T, Yasuda H, Terai H, Kagiwada H, Hamamoto J, Ebisudani T, Kobayashi K, Masuzawa K, Ikemura S, Kawada I, Hayashi Y, Fukui K, Horimoto K, et al. IGF2 autocrine-mediated IGF1R activation is a clinically relevant mechanism of osimertinib resistance in lung cancer. Mol Cancer Res. 2020; 18:549-559. https://doi. org/10.1158/1541-7786.MCR-19-0956. [PubMed]

24. Hiraoka A, Kumada T, Kariyama K, Takaguchi K, Atsukawa M, Itobayashi E, Tsuji K, Tajiri K, Hirooka M, Shimada N, Shibata H, Ishikawa T, Ochi H, et al, and Real-life Practice Experts for HCC (RELPEC) Study Group, and HCC 48 Group (hepatocellular carcinoma experts from 48 clinics in Japan). Clinical features of lenvatinib for unresectable hepatocellular carcinoma in real-world conditions: Multicenter analysis. Cancer Med. 2019; 8:137-146. https:// doi.org/10.1002/cam4.1909. [PubMed]
25. Baumgartner RN, Koehler KM, Romero L, Garry PJ. Serum albumin is associated with skeletal muscle in elderly men and women. Am J Clin Nutr. 1996; 64:552-558. https://doi. org/10.1093/ajen/64.4.552. [PubMed]

26. Visser M, Kritchevsky SB, Newman AB, Goodpaster BH, Tylavsky FA, Nevitt MC, Harris TB. Lower serum albumin concentration and change in muscle mass: the Health, Aging and Body Composition Study. Am J Clin Nutr. 2005; 82:531-537. https://doi.org/10.1093/ajen/82.3.531. [PubMed]

27. Gariballa S, Alessa A. Association between nutritional blood-based biomarkers and clinical outcome in sarcopenia patients. Clin Nutr ESPEN. 2018; 25:145-148. https://doi. org/10.1016/j.clnesp.2018.03.002. [PubMed]

28. Ueshima K, Nishida N, Hagiwara S, Aoki T, Minami T, Chishina H, Takita M, Minami Y, Ida H, Takenaka M, Sakurai T, Watanabe T, Morita M, et al. Impact of baseline ALBI grade on the outcomes of hepatocellular carcinoma patients treated with lenvatinib: A multicenter study. Cancers (Basel). 2019; 11:952. https://doi.org/10.3390/ cancers11070952. [PubMed]

29. Galle PR, Forner A, Llovet JM, Mazzaferro V, Piscaglia F, Raoul JL, Schirmacher P, Vilgrain V, and European Association for the Study of the Liver. EASL Clinical Practice Guidelines: Management of hepatocellular carcinoma. J Hepatol. 2018; 69:182-236. https://doi. org/10.1016/i.jhep.2018.03.019. [PubMed]

30. Lencioni R, Llovet JM. Modified RECIST (mRECIST) assessment for hepatocellular carcinoma. Semin Liver Dis. 2010; 30:52-60. https://doi.org/10.1055/s-0030-1247132. [PubMed]

31. Oken MM, Creech RH, Tormey DC, Horton J, Davis TE, McFadden ET, Carbone PP. Toxicity and response criteria of the Eastern Cooperative Oncology Group. Am J Clin Oncol. 1982; 5:649-655. https://doi.org/10.1097/00000421198212000-00014. [PubMed]

32. Llovet JM, Bru C, Bruix J. Prognosis of hepatocellular carcinoma: the BCLC staging classification. Semin Liver Dis. 1999; 19:329-338. https://doi.org/10.1055/s-2007-1007122. [PubMed]

33. Kudo M, Kitano M, Sakurai T, Nishida N. General rules for the clinical and pathological study of primary liver cancer, nationwide follow-up survey and clinical practice guidelines: The Outstanding Achievements of the Liver Cancer Study Group of Japan. Dig Dis. 2015; 33:765-770. https://doi.org/10.1159/000439101. [PubMed]

34. Johnson PJ, Berhane S, Kagebayashi C, Satomura S, Teng M, Reeves HL, O’Beirne J, Fox R, Skowronska A, Palmer D, Yeo W, Mo F, Lai P, et al. Assessment of liver function in patients with hepatocellular carcinoma: a new evidencebased approach-the ALBI grade. J Clin Oncol. 2015; 33:550-558. https://doi.org/10.1200/JCO.2014.57.9151. [PubMed] 